\title{
Comparação da eficácia dos corticosteroides nos pacientes com hérnia discal extrusa submetidos a bloqueio radicular transforaminal
}

\author{
Comparison of the effectiveness of corticosteroids in patients with extrusus discal hernia \\ submitted to transforaminal radicular block \\ Comparación de la eficacia de los corticosteroides en pacientes con hernia de disco \\ extrusus presentado en el bloque radicular transforaminal
}

Vinícius Genelhu Abreu de Souza Cóbe ${ }^{1}$, Otávio Montovanelli Monteiro ${ }^{1 *}$, Jackson Oliveira da Cruz Júnior $^{2}$, Ricardo Sturzeneker Cerqueira Lima ${ }^{1}$, Diego Barcellos Caldas ${ }^{1}$, Eduardo Correa Damázio ${ }^{1}$, José Lucas Batista Júnior ${ }^{1}$, Igor Machado Cardoso ${ }^{1}$, Joelmar Cezar de Almeida ${ }^{1}$, Chárbel Jacob Júnior ${ }^{1}$.

\section{RESUMO}

Objetivo: Comparar a eficácia de diferentes corticosteroides (dexametasona, betametasona e triancinolona) utilizados na técnica de bloqueio do ramo troncular, por meio da avaliação da melhora do quadro álgico, em pacientes com lombociatalgia. Métodos: 60 pacientes maiores de 18 anos, com queixa de lombociatalgia refratária ao tratamento conservador, diagnosticados com hérnia de disco lombar foraminal extrusa por meio do exame de ressonância magnética, sem critérios de instabilidade vertebral em radiografias dinâmicas da coluna lombar foram divididos em 3 grupos de 20 indivíduos cada. O Grupo 1 recebeu dexametasona, o Grupo 2 betametasona e o Grupo 3 triancinolona. Todos os pacientes responderam à escala visual analógica nos períodos de pré-bloqueio e com 1, 6 e 12 meses após a realização do mesmo para avaliação de dor. Resultados: Foi observado melhora significativa da dor após a realização do bloqueio nos 3 grupos, não havendo diferença estatística entre os fármacos. Conclusão: $O$ bloqueio do ramo troncular é uma técnica eficaz no tratamento das lombociatalgias, contudo, não existe diferença na eficácia dos três fármacos estudados. Sendo assim, a escolha do corticosteroide deve ser feita de forma individualizada, levando-se em conta fatores não avaliados nesse estudo, como disponibilidade, custo e reações adversas.

Palavras-chave: Dor lombar, Corticosteroides, Bloqueio nervoso, Deslocamento do disco intervertebral.

\begin{abstract}
Objective: To compare the efficacy of different corticosteroids (dexamethasone, betamethasone and triamcinolone) used in the trunk branch block technique, by assessing the improvement of pain in patients with low back pain. Methods: 60 patients over 18 years old, complaining of low back pain refractory to conservative treatment, diagnosed with extruded foraminal lumbar disc herniation by means of magnetic resonance imaging, without vertebral instability criteria in dynamic lumbar spine radiographs were divided into 3 groups of 20 individuals each. Group 1 received dexamethasone, Group 2 betamethasone and Group 3 triamcinolone. All patients responded to the visual analogue scale in the pre-blocking periods and at 1, 6 and 12 months after performing the same for pain assessment. Results: A significant improvement in pain was observed after the blockade in all 3 groups, with no statistical difference between the drugs. Conclusion: Blockade of the trunk branch is an effective technique in the treatment of lumbar sciatica, however, there is no difference in the effectiveness of the three drugs studied. Therefore, the choice of corticosteroids should be made individually, considering factors not evaluated in this study, such as availability, cost and adverse reactions.
\end{abstract}

Keywords: Low back pain, Adrenal cortex hormones, Nerve block, Intervertebral disc displacement.

${ }^{1}$ Santa Casa de Misericórdia de Vitória, Vitória - ES. *E-mail: otaviomm88@gmail.com

2 Vila Velha Hospital, Vila Velha - ES. 


\section{RESUMEN}

Objetivo: Comparar la eficacia de los diferentes corticoesteroides (dexametasona, betametasona y triamcinolona) utilizados en la técnica de bloqueo de ramas del tronco, evaluando la mejora del dolor en pacientes con dolor lumbar. Métodos: 60 pacientes mayores de 18 años, quejándose de lumbalgia refractaria a tratamiento conservador, diagnosticados con hernia de disco lumbar foraminal extruido por medio de imágenes de resonancia magnética, sin criterios de inestabilidad vertebral en radiografías dinámicas de columna lumbar se dividieron en 3 grupos de 20 individuos cada uno. El grupo 1 recibió dexametasona, el grupo 2 betametasona y el grupo 3 triamcinolona. Todos los pacientes respondieron a la escala analógica visual en los períodos de prebloqueo y a los 1, 6 y 12 meses después de la realización de la evaluación del dolor. Resultados: Se observó una mejora significativa en el dolor después del bloqueo en los 3 grupos, $\sin$ diferencias estadísticas entre los fármacos. Conclusión: El bloqueo de la rama del tronco es una técnica efectiva en el tratamiento de la ciática lumbar, sin embargo, no hay diferencia en la efectividad de los tres fármacos estudiados. Por lo tanto, la elección de los corticosteroides debe hacerse individualmente, teniendo en cuenta los factores no evaluados en este estudio, como la disponibilidad, el costo y las reacciones adversas.

Palabras clave: Dolor de la region lumbar, Corticoesteroides, Bloqueo nervioso, Desplazamiento del disco intervertebral.

\section{INTRODUÇÃO}

A lombalgia, definida como dor e desconforto localizados entre a margem costal e a prega glútea inferior, está entre as 10 principais causas pelas quais os indivíduos procuram atendimento médico (ANDERSON JAD, 1977; ALMEIDA DC e KRAYCHETE DC, 2017). Estima-se que $80 \%$ das pessoas apresentarão dor lombar em algum momento de suas vidas (ALMEIDA DC e KRAYCHETE DC, 2017).

Em $60 \%$ desses casos ocorre a lombociatalgia, quando há dor irradiada para o membro inferior, que pode ser de origem radicular, por compressão ou referida (STUMP PRNAG, et al., 2016). Os mecanismos fisiopatológicos da ciatalgia ainda são pouco conhecidos, no entanto acredita-se que a fisiopatologia da dor radicular tenha origem devido à deformação mecânica e lesão inflamatória mediada por interferon-alfa e outras citocinas (RYDEVIK B, et al., 1984; MULLEMAN D, et al., 2006).

A prevalência de hérnia de disco lombar na população é calculada entre 2\% a 3\% (ZANON IB, et al., 2015). Ela ocorre quando há extravasamento do conteúdo do núcleo pulposo do disco intervertebral através do seu anel fibroso (VIALLE LR, et al., 2010).

Tal alteração degenerativa pode causar compressão das raízes nervosas levando a ciatalgia (ZANON IB, et al., 2015). Além disso, mediadores inflamatórios do núcleo pulposo herniado em contato com a raiz nervosa também contribuem para a etiologia da dor ciática (ZANON IB, et al., 2015).

Atualmente, é consenso na literatura que o tratamento inicial da lombociatalgia por herniação discal deve ser conservador, incluindo repouso por curto período, medicamentos, fisioterapia, alongamento, fortalecimento muscular e reeducação postural, entre outros, com taxa de sucesso de cerca de $90 \%$ dos casos (SAAL JA, et al., 1990; SAAL JÁ e SAAL JS, 1989). No entanto, a presença de déficit neurológico progressivo, síndrome da cauda equina ou persistência da hérnia e do quadro clínico podem indicar a necessidade de tratamento cirúrgico (POSTACCHINI F, 1999; VIALLE LR, et al., 2010).

Porém, levando-se em conta as mudanças inflamatórias locais e seu papel importante como fatores contribuintes para a dor radicular na hérnia discal, e alternativamente ao tratamento cirúrgico, tem-se utilizado como técnica minimamente invasiva a realização do Bloqueio de Ramo Transforaminal (BRT). Neste procedimento, muito embora não haja remoção do material herniado, há possibilidade de melhora significativa na qualidade de vida permitindo a continuação do tratamento conservador (LESLIE NG, et al., 2005; SAYEGH FE, et al., 2009). 
A técnica supracitada baseia-se no uso de injeções de anestésicos locais e corticosteroides. Este é um procedimento menos invasivo e com menor morbimortalidade, quando comparado ao procedimento cirúrgico, e vem sendo utilizado com o objetivo de aliviar os sintomas dolorosos, melhorando a qualidade de vida, diminuindo $o$ afastamento das atividades produtivas e reduzindo o índice de procedimentos cirúrgicos em todo o mundo (MANSON NA, et al., 2013).

Os riscos mais comuns descritos com esta técnica são de infecção, hematoma peridural, lesão nervosa, reações alérgicas, insuficiência adrenal, edema, pneumotórax, anestesia subaracnóidea total e dor lombar após a punção (COLLIGHAN N e GUPTA S, 2010).

Apesar disso, a técnica apresenta índices muito baixos de complicações, sendo o seu uso seguro, sobretudo, quando respeitadas as suas contraindicações, tais como, hipersensibilidade às substâncias utilizadas, infecção sistêmica ou no local da punção, neoplasia local e coagulopatia (TONKOVICHQUARANTA LA e WINKLER SR., 2000; ZANON IB, et al., 2015; ALVES TCA, et al., 2015).

Deve-se tomar maior precaução também em pacientes portadores de insuficiência cardíaca congestiva, diabetes mellitus descompensado, imunossupressão e nas gestantes (ALVES TCA, et al., 2015). Devemos salientar, porém, que as suas contraindicações não diferem tanto das contraindicações das técnicas cirúrgicas convencionais.

Metilprednisolona, triancinolona, betametasona e dexametasona são corticoides que podem ser utilizados nas injeções peridurais. Anteriormente a hidrocortisona também era utilizada, contudo, devido a maior duração de efeito e potência das demais drogas, acabou sendo substituída (TONKOVICH-QUARANTA LA e WINKLER SR, 2000).

Outro motivo pelo qual a hidrocortisona deixou de ser utilizada é o fato de possuir maior efeito mineralocorticóide, que pode levar a retenção de sódio e aumento da pressão arterial (TONKOVICHQUARANTA LA e WINKLER SR, 2000). Tais efeitos também podem ocorrer com maior frequência quando se utiliza a prednisolona (TONKOVICH-QUARANTA LA e WINKLER SR, 2000).

Estudos demonstraram maior eficácia apenas em curto prazo dos corticoides particulados, como a Betametasona (BET) e Triancinolona (TRI), se comparados aos não particulados, como a Dexametasona (DEX) (KENNEDY DJ, et al., 2014). Todavia, a literatura carece de evidências que comparem a eficácia dos corticosteroides em longo prazo, de forma a guiar a escolha dos mesmos no bloqueio epidural.

Diante do exposto, o presente trabalho teve por objetivo a realização de uma análise comparativa entre os três corticosteroides supracitados, por meio da avaliação da melhora do quadro álgico após o procedimento, a fim de possibilitar melhor orientação quanto à escolha da terapia a ser realizada nos pacientes portadores de dor radicular.

\section{MÉTODOS}

O estudo realizado trata-se de um ensaio clínico comparativo randomizado duplo cego, a partir dos dados coletados por meio de Ressonância Nuclear Magnética (RNM) e questionários, nos diferentes períodos do estudo.

O estudo foi realizado após a autorização do Comitê de Ética em Pesquisa (CEP) em Seres Humanos da instituição onde as cirurgias foram realizadas. O mesmo foi submetido e veiculado à Plataforma Brasil.

A população foi composta por 60 pacientes. Estes foram atendidos pelo grupo de cirurgia de coluna de um único hospital, localizado em um município do Espírito Santo, durante os anos de 2014 a 2018.

Foram incluídos neste estudo pacientes maiores de dezoito anos diagnosticados com hérnia de disco lombar foraminal extrusa por meio do exame de RNM, que apresentavam quadro de dor radicular refratária ao tratamento conservador; nos quais não foram observados critérios de instabilidade vertebral avaliados por meio de radiografias dinâmicas lombares; e que concordaram com o Termo de Consentimento Livre e Esclarecido (TCLE) do projeto. 
Para aferir se havia presença de instabilidade vertebral, foram avaliadas as radiografias dinâmicas em perfil da coluna lombar. Os critérios de instabilidade utilizados foram a translação entre os corpos vertebrais maior que $3 \mathrm{~mm}$ e a angulação dos platôs vertebrais maior do que $18^{\circ}$ (WHITE AA e PANJABI MM, 1990; ZANON IB, et al., 2015).

Não fizeram parte deste estudo os pacientes que não apresentavam quadro de compressão radicular ou aqueles que tiveram recorrência do quadro clínico após procedimento realizado previamente, sendo encaminhados para novo BRT ou para o tratamento cirúrgico convencional.

Está assegurado o sigilo das informações extraídas dos prontuários e de exames de imagem mediante a não identificação nominal dos pacientes, os quais foram identificados numericamente procedidos pela letra $P$ (P 01, P 02, etc.). As informações obtidas ficarão sob tutela do pesquisador responsável durante o período de cinco anos.

Os pacientes foram convidados por meio de uma carta, entregue no momento em que compareceram para a avaliação clínica com a confirmação diagnóstica, contendo as explicações da pesquisa para a leitura e assinatura do TCLE.

Aqueles que se enquadraram nos critérios de inclusão e que não atenderam aos critérios de exclusão, responderam a Escala analógica visual (VAS) para avaliação do quadro álgico, sendo utilizado instrumento semelhante a uma régua, com medida de $10 \mathrm{~cm}$ e numerado de 0 a 10 , de modo que 0 representa a ausência de dor e 10 retrata a "dor insuportável" ou "a pior dor imaginável" (SCOTT J e HUSKISSON EC, 1976).

Após respondido o questionário supracitado, os pacientes foram submetidos ao BRT. Neste procedimento, foram utilizadas três drogas da classe dos corticosteroides: Dexametasona, Betametasona e Triancinolona. Todos os participantes receberam o volume de $5 \mathrm{ml}$ de solução contendo o corticóide e o anestésico cloridrato de bupivacaína na apresentação $0,5 \%$ sem vasoconstrictor. Os pacientes foram separados em três grupos de vinte indivíduos cada um.

O grupo 1 recebeu o composto Dexametasona na dose de $2,5 \mathrm{~mL}(4 \mathrm{mg} / \mathrm{mL})+1 \mathrm{~mL}(2 \mathrm{mg} / \mathrm{mL})+1,5 \mathrm{~mL}$ de anestésico. Já os pacientes do grupo 2 receberam o composto Betametasona na dose de $3 \mathrm{~mL}(4 \mathrm{mg} / \mathrm{mL})$ $+2 \mathrm{~mL}$ de anestésico. O grupo 3, por sua vez, recebeu o composto Triancinolona na dose de $2 \mathrm{~mL}(20 \mathrm{mg} / \mathrm{mL})$ $+3 \mathrm{mg}$ de anestésico (Tabela 1).

Tabela 1 - Característica da amostra de acordo com o grupo.

\begin{tabular}{|c|c|c|c|c|c|c|c|}
\hline \multirow{2}{*}{\multicolumn{2}{|c|}{ Amostra }} & \multicolumn{6}{|c|}{ Composto } \\
\hline & & \multicolumn{2}{|l|}{ Dex } & \multicolumn{2}{|c|}{ Bet } & \multicolumn{2}{|l|}{ Tri } \\
\hline \multirow{3}{*}{ Sexo } & & & & & & & \\
\hline & $\mathrm{F}$ & 14 & 70,0 & 12 & 60,0 & 10 & 50,0 \\
\hline & $\mathrm{M}$ & 6 & 30,0 & 8 & 40,0 & 10 & 50,0 \\
\hline \multirow{4}{*}{ Sintoma } & Ciática d & 4 & 20,0 & 1 & 5,0 & 0 & 0,0 \\
\hline & Ciática e & 4 & 20,0 & 2 & 10,0 & 0 & 0,0 \\
\hline & Lombociatalgia d & 9 & 45,0 & 9 & 45,0 & 13 & 65,0 \\
\hline & Lombociatalgia e & 3 & 15,0 & 8 & 40,0 & 7 & 35,0 \\
\hline \multirow{3}{*}{ Composto } & Bet & 0 & 0,0 & 20 & 100,0 & 0 & 0,0 \\
\hline & Dex & 20 & 100,0 & 0 & 0,0 & 0 & 0,0 \\
\hline & Tri & 0 & 0,0 & 0 & 0,0 & 20 & 100,0 \\
\hline
\end{tabular}

Legenda: F: feminino; M: masculino; d: direita; e: esquerda; Bet: betametasona; Dex: dexametasona; Tri: triancinolona; №: número. Fonte: Cóbe VGAS, et al., 2020.

As medicações foram administradas de forma randomizada, sem conhecimento de qual droga foi aplicada em cada paciente. Um recipiente lacrado, preparado antes do início dos procedimentos, continha 60 envelopes ( 20 de cada grupo). No dia em que o paciente seria submetido ao bloqueio epidural um dos membros da equipe fazia o sorteio de 1 envelope para definir de qual grupo ele faria parte. 
Foi aplicado novamente, de forma seriada (com 1, 6 e 12 meses após o procedimento) o questionário de avaliação de dor, com o objetivo de se comparar os resultados entre si, e destes com os dados obtidos anteriormente ao procedimento de BRT.

Após análise dos dados, foi verificado qual droga apresentava melhor resposta terapêutica quando administrada por meio de BRT. A análise dos dados foi composta de uma análise descritiva, onde a distribuição da variável Escala de Dor (quantitativa) foi avaliada mediante a determinação de suas medidas de posição central e variabilidade (mediana, média e desvio padrão).

Para os cruzamentos da Escala de Dor entre os grupos foi utilizado o teste não paramétrico de KruskalWallis, e para a comparação da Escala de Dor entre os momentos foi realizado o teste não paramétrico de Friedman. Foram utilizados testes não paramétricos pois a normalidade dos dados foi rejeitada segundo teste de Shapiro-Wilk.

Toda inferência estatística foi realizada considerando o Intervalo de Confiança (IC) para os dados analisados de $95 \%$ ( $p \leq 0,05)$. Os dados foram organizados em planilha do software Microsoft Excel $\Theta 365$, incluído no Office 365 e analisados pelo software SPSS versão 23.

\section{RESULTADOS}

Não ocorreram mudanças de metodologia durante o estudo. O perfil da idade, sexo, nível do bloqueio na coluna lombar e queixa clínica dos pacientes avaliados não apresentaram grandes variações. Respectivamente os grupos 1, 2 e 3 apresentaram valores médios pré-bloqueio para a VAS de 7,90, 8,55 e 8,60. Esses valores foram semelhantes estatisticamente.

Os pacientes do Grupo 1, que receberam solução contendo dexametasona, apresentaram pontuação média de 4,90 com 1 mês após procedimento, aos 6 meses 5,55 e aos 12 meses 4,70. No Grupo 2 (Betametasona) a VAS média foi de 4,75 no 1ํㅡês pós-op, 6,05 aos 6 meses e 5,45 12 meses após a cirurgia. Já o Grupo 3, em que foi administrada a triancinolona, apresentou respectivamente VAS média de 6,55, 6,25 e 5,30 nos momentos 1 mês, 6 meses e 12 meses (Tabela 2).

Tabela 2 - Escala visual analógica (VAS).

\begin{tabular}{cccccccc}
\hline Grupos & Momentos & $\mathbf{N}$ & $\begin{array}{c}\text { Menor } \\
\text { Valor }\end{array}$ & $\begin{array}{c}\text { Maior } \\
\text { Valor }\end{array}$ & Mediana & Média & $\begin{array}{c}\text { Desvio- } \\
\text { Padrão }\end{array}$ \\
\hline \multirow{3}{*}{ Dexametasona } & Pré-op & 20 & 4,00 & 10,00 & 8,00 & 7,90 & 1,65 \\
& 1 mês & 20 & 0 & 10,00 & 5,50 & 4,90 & 3,45 \\
& 6 meses & 20 & 0 & 10,00 & 5,00 & 5,55 & 3,38 \\
Betametasona & 12 meses & 20 & 0 & 10,00 & 4,50 & 4,70 & 2,87 \\
& Pré-op & 20 & 5,00 & 10,00 & 9,00 & 8,55 & 1,36 \\
& 1 mês & 20 & 0 & 10,00 & 5,50 & 4,75 & 3,24 \\
& 6 meses & 20 & 0 & 10,00 & 7,00 & 6,05 & 2,70 \\
Triancinolona & 12 meses & 20 & 0 & 9,00 & 7,00 & 5,45 & 3,09 \\
\hline & Pré-op & 20 & 7,00 & 10,00 & 9,00 & 8,60 & 1,27 \\
& 1 mês & 20 & 1,00 & 10,00 & 7,00 & 6,55 & 2,39 \\
& 6 meses & 20 & 0 & 10,00 & 6,50 & 6,25 & 2,47 \\
\hline
\end{tabular}

Legenda: Pré-op: pré-operatório; N: tamanho da amostra. Fonte: Cóbe VGAS, et al., 2020.

Para cada um dos grupos foi encontrada diferença estatisticamente significante na Escala de Dor entre os momentos pré-operatório e os demais momentos pós-operatórios ( $p$-valor $<0,05$ ). No entanto, não houve diferença estatisticamente significante na escala de dor entre os momentos 1,6 e 12 meses. Logo, foi observado que todos os três grupos se comportam de maneira semelhante. Sendo assim, pode-se dizer que no pré-operatório se encontram os maiores valores da Escala de Dor (Tabela 3). 
Tabela 3 - Estatísticas descritivas da Escala de Dor segundo Momentos e resultados do teste de Friedman.

\begin{tabular}{|c|c|c|c|c|c|c|}
\hline \multirow{2}{*}{ Grupos } & \multirow{2}{*}{ Momentos } & \multirow{2}{*}{ Mediana } & \multirow{2}{*}{ Média } & \multirow{2}{*}{$\begin{array}{l}\text { Desvio- } \\
\text { padrão }\end{array}$} & \multicolumn{2}{|c|}{$\begin{array}{l}\text { Teste de } \\
\text { Friedman }\end{array}$} \\
\hline & & & & & $\begin{array}{l}\text { Postos } \\
\text { Médios }\end{array}$ & p-valor \\
\hline \multirow{4}{*}{ Dexametasona } & Pré-op & 8,00 & 7,90 & 1,65 & $3,33 \mathrm{~A}$ & \multirow{4}{*}{0,008} \\
\hline & 1 mês & 5,50 & 4,90 & 3,45 & $2,13_{\mathrm{B}}$ & \\
\hline & 6 meses & 5,00 & 5,55 & 3,38 & $2,38 \mathrm{~B}$ & \\
\hline & 12 meses & 4,50 & 4,70 & 2,87 & $2,18 \mathrm{~B}$ & \\
\hline \multirow{4}{*}{ Betametasona } & Pré-op & 9,00 & 8,55 & 1,36 & $3,43 \mathrm{~A}$ & \multirow{4}{*}{0,001} \\
\hline & 1 mês & 5,50 & 4,75 & 3,24 & 1,93 B & \\
\hline & 6 meses & 7,00 & 6,05 & 2,70 & $2,43_{B}$ & \\
\hline & 12 meses & 7,00 & 5,45 & 3,09 & $2,23 \mathrm{~B}$ & \\
\hline \multirow{4}{*}{ Triancinolona } & Pré-op & 9,00 & 8,60 & 1,27 & $3,45 \mathrm{~A}$ & \multirow{4}{*}{0,001} \\
\hline & 1 mês & 7,00 & 6,55 & 2,39 & $2,38_{B}$ & \\
\hline & 6 meses & 6,50 & 6,25 & 2,47 & 2,23 в & \\
\hline & 12 meses & 5,50 & 5,30 & 2,90 & $1,95_{B}$ & \\
\hline
\end{tabular}

Legenda: Pré-op: pré-operatório. *Letras diferentes denotam diferenças estatisticamente significantes. Fonte: Cóbe VGAS, et al., 2020.

Como observado, não foi obtido $p$-valor $<0,05$. Desse modo, constata-se que não houve diferença estatisticamente significante entre a capacidade dos fármacos em diminuir a dor na comparação da Escala de Dor entre os grupos, ou seja, a capacidade dos três fármacos em diminuir a dor do paciente foi semelhante (Tabela 4).

Tabela 4 - Teste de comparação entre os grupos. Estatísticas descritivas da Escala de Dor segundo grupos e resultados do teste de Kruskal-Wallis.

\begin{tabular}{cccccc}
\hline Momentos & Grupos & Mediana & Média & $\begin{array}{c}\text { Desvio- } \\
\text { Padrão }\end{array}$ & $\begin{array}{c}\text { p-valor } \\
\text { (Kruskal- } \\
\text { Wallis) }\end{array}$ \\
\hline \multirow{2}{*}{ Pré-op } & Dexametasona & 8,00 & 7,90 & 1,65 & \\
& Betametasona & 9,00 & 8,55 & 1,36 & 0,308 \\
& Triancinolona & 9,00 & 8,60 & 1,27 & \\
\hline \multirow{2}{*}{$\mathbf{1}$ mês } & Dexametasona & 5,50 & 4,90 & 3,45 & \\
& Betametasona & 5,50 & 4,75 & 3,24 & 0,141 \\
& Triancinolona & 7,00 & 6,55 & 2,39 & \\
\hline \multirow{2}{*}{$\mathbf{6}$ meses } & Dexametasona & 5,00 & 5,55 & 3,38 & \\
& Betametasona & 7,00 & 6,05 & 2,70 & 0,797 \\
& Triancinolona & 6,50 & 6,25 & 2,47 & \\
\hline \multirow{2}{*}{$\mathbf{1 2}$ meses } & Dexametasona & 4,50 & 4,70 & 2,87 & \\
& Betametasona & 7,00 & 5,45 & 3,09 & 0,658 \\
& Triancinolona & 5,50 & 5,30 & 2,90 & \\
\hline
\end{tabular}

Legenda: Pré-op: pré-operatório. Fonte: Cóbe VGAS, et al., 2020.

\section{DISCUSSÃO}

Foi relatado que a demora na realização da cirurgia, nos casos de lombociatalagia em que está indicado o tratamento cirúrgico, pode piorar os sintomas, levando à cronicidade do quadro álgico por meio da ativação do sistema modulador da dor e efeito de memória. Alguns estudos mostram taxas de cronificação de até $44 \%$ (ITZ CJ, et al., 2013; STUMP PRNAG, et al., 2016). 
Os casos crônicos são aqueles em que o tempo de evolução é maior do que 3 meses (AIRAKSINEN O, et al., 2006; ALMEIDA DC e KRAYCHETE DC, 2017). Sendo assim, é essencial que os pacientes tenham acesso a acompanhamento inicial e a tratamento adequado da lombalgia aguda (STUMP PRNAG, et al., 2016).

Nesse âmbito, a possibilidade de tratamento com o bloqueio radicular utilizando corticóide vem ganhando cada vez mais importância, tendo em vista que se trata de um procedimento seguro e de menor custo (ZANON IB, et al., 2015). Já foi relatado que a injeção transforaminal de corticóide em pacientes com dor ciática reduziu em $56 \%$ a quantidade de procedimentos invasivos em pacientes com quadro crônico e disfunção, sendo eficaz no alívio da dor e na recuperação da funcionalidade mesmo em pacientes com período prolongado de sintomas e retardo do início do tratamento (MANSON NA, et al., 2013).

Justifica-se assim a sua utilização, uma vez que oferece menores riscos de complicação e menor tempo de internação hospitalar, tendo em vista a alta hospitalar precoce após o procedimento, além de uma redução de custos e da incidência de cronificação dos casos. Por esses motivos, a utilização da técnica de BRT tem sido cada vez mais frequente. Estudos mostram que nos Estados Unidos da América, a quantidade deste procedimento teve um aumento de $665 \%$ no período de 2000 a 2011 (DENIS I, et al., 2015).

Existem diversos substratos de ação descritos para o efeito anti-inflamatório dessas drogas. Uma ação importante é o bloqueio da fosfolipase A2 com inibição da liberação do ácido aracdônico, reduzindo, dessa maneira, a produção de ciclo-oxigenases e lipo-oxigenases com consequente redução da formação de prostaglandinas, tromboxanos e leucotrienos, mediadores com importante participação na cascata inflamatória e na fisiopatologia da dor (COLLIGHAN N e GUPTA S, 2010). Verificou-se, ainda, em modelos experimentais, que a betametasona pode diminuir a expressão da substância $P$, sugerindo um possível efeito direto dos corticosteroides sobre os mediadores da dor (COLLIGHAN N e GUPTA S, 2010).

Ainda, independentemente de sua ação anti-inflamatória, os corticoides parecem exercer também efeito do tipo anestésico, bloqueando a condução das fibras nociceptivas $C$, além de inibirem a agregação leucocitária, o que causaria interrupção da cascata de inflamação no tecido afetado (JOHANSSON A, et al., 1990).

O corticóide pode ser diluído em solução fisiológica isotônica para a aplicação de injeção peridural, contudo é mais cômodo para o paciente quando diluída em anestésico local (SPACCARELLI KC, 1996). Existe vantagem teórica na utilização dos anestésicos locais como diluentes pois pode ser rompido o ciclo espamoisquemia-dor, levando ao relaxamento muscular. Além disso, causa bloqueio sensitivo que permite averiguar de maneira mais acertiva o local de aplicação (CANNON DT e APRILL CN, 2000).

Há, porém, uma grande divergência quanto ao melhor fármaco a ser utilizado no BRT. Um estudo comparou a injeção de $80 \mathrm{mg}$ de TRI com $8 \mathrm{mg}$ de DEX, obtendo uma maior redução da VAS com o grupo da TRI no período de duas semanas, quando comparado à DEX (2.87 versus $1,21-p=0,037)$ (CANNON DT e APRILL CN, 2000). Outra pesquisa comparou $40 \mathrm{mg}$ de TRI e 7,5 mg de DEX no período de um mês, e evidenciou uma redução mais significativa da VAS nos pacientes nos quais foi utilizada a TRI, quando comparado aos pacientes em uso da DEX (71\% versus $40 \%-p=0,000)$. Entretanto, não houve diferença ao avaliar o questionário Oswestry (PARK CH, et al., 2010).

Foram comparados também $80 \mathrm{mg}$ de TRI, $12 \mathrm{mg}$ de BET, e $10 \mathrm{mg}$ de DEX, demonstrando no período de dois meses superioridade da DEX no alívio da dor (EL-YAHCHOUCHI C, et al., 2013). A utilização de $60 \mathrm{mg}$ de TRI foi comparada a $15 \mathrm{mg}$ de DEX no período de seis meses de seguimento, não mostrando diferença entre as drogas (KENNEDY DJ, et al., 2014).

Ocorre também grande variação em relação às doses de corticosteroides utilizadas em diversos trabalhos. Usualmente, são empregues entre $40 \mathrm{mg}$ e $80 \mathrm{mg}$ de triancinolona, $6 \mathrm{mg}$ a $12 \mathrm{mg}$ de betametasona, $8 \mathrm{mg}$ a $12 \mathrm{mg}$ de dexametasona (ADAM A, et al., 2020). Em nosso estudo procuramos respeitar esses limites. Frente às divergências encontradas na literatura e à necessidade de complementar o entendimento acerca da melhor escolha dos corticosteroides no BRT, este trabalho teve como modelo um seguimento a longo prazo, com uma amostra significativa, dividida de forma homogênea e randomizada, que garante a relevância estatística dos dados obtidos pelo mesmo. 
Para avaliar a resposta terapêutica dos tratamentos realizados foi utilizada a Escala Visual Analógica (VAS) no pré-operatório e em 3 momentos após a realização do procedimento (SCOTT J e HUSKISSON EC, 1976). A escolha desse método ocorreu devido a uma soma de fatores. Um deles foi a facilidade de aplicação do questionário pela equipe e a compreensibilidade por parte dos avaliados (BOONSTRA AM, et al., 2008).

O tempo necessário para aplicar o mesmo geralmente é menor do que 1 minuto (HAWKER GA, et al., 2011). Outro motivo foi o fato de que é uma escala confiável e validada para avaliação da dor de origem musculoesquelética (HAWKER GA, et al., 2011). Além do mais, trata-se de um consagrado método utilizado em diversos trabalhos para aquilatar a percepção da dor (LESLIE NG, et al., 2005; PINHEIRO J, et al., 2011; ZANON IB, et al., 2015).

O presente estudo evidenciou que desde a avaliação de um mês até o seguimento de doze meses, em todos os grupos, obteve-se melhora do quadro álgico, conforme exposto na Tabela 3. Entretanto, ao comparar os três grupos, não foi observada superioridade com diferença estatística quanto à eficácia em redução da dor entre os fármacos utilizados, conforme descrito na Tabela 4.

Resta, portanto, encontrar características peculiares de cada um dos fármacos que auxiliem na escolha da droga por parte do profissional médico, devendo ser levados em conta a acessibilidade, os custos e os efeitos adversos relacionados de cada droga. Dentre as drogas utilizadas, a triancinolona apresenta geralmente o maior custo para aquisição, devendo ser evitada quando o objetivo for preservar financeiramente a instituição de saúde ou o paciente. Já a betametasona e a dexametasona apresentam menores custos e preços similares.

Quanto aos efeitos adversos, alguns relatos de caso demonstraram a ocorrência de eventos embólicos e isquêmicos em medula espinhal relacionados ao uso de corticosteroides particulados, que apesar de serem raros, devem ser considerados, já que a Dexametasona (corticosteroide não particulado), apresenta eficácia terapêutica similar aos particulados (GHARIBO C, et al., 2009).

A metilprednisolona e a triancinolona são drogas preferencialmente utilizadas em injeções peridurais por apresentarem boa potência antiinflamatória além de baixos efeitos adversos e mineralocorticoides, quando comparadas a outros corticosteroides (TONKOVICH-QUARANTA LA e WINKLER SR, 2000). Todavia, similarmente aos estudos supracitados, nota-se que ainda há necessidade da realização de pesquisas de maior proporção, a fim de determinar de maneira mais clara o fármaco de escolha no BRT.

\section{CONCLUSÃO}

O Bloqueio de Ramo Troncular é uma importante ferramenta no tratamento da ciatalgia. Trata-se de um procedimento capaz de diminuir a dor e melhorar a função da maioria dos pacientes, permitindo que eles realizem de maneira mais eficaz as medidas conservadoras de tratamento, como fisioterapia e fortalecimento muscular. Além disso, apresenta baixo índice de complicações e diminuem a necessidade de realização de tratamentos cirúrgicos, mais custosos e com maior morbidade. No presente estudo houve melhora significativa no alívio da dor em cada um dos grupos, tal fato corrobora para a maior utilização da técnica. Contudo, quando comparados os fármacos entre si, não houve diferença estatística da eficácia entre eles utilizando-se como parâmetro a redução da pontuação na escala visual analógica de dor, obtendo-se resultados semelhantes para todos os grupos. Isto posto, recomenda-se que a escolha do fármaco a ser utilizado deva ser feita de forma individualizada, levando em consideração sua composição, custo, efeitos adversos e disponibilidade. Sendo, portanto, necessários novos estudos que incluam em sua metodologia uma análise desses fatores, a fim de elucidar melhor a escolha do fármaco no bloqueio do ramo troncular.

\section{REFERÊNCIAS}

1. ADAM A, et al. Grainger \& Allison's Diagnostic Radiology A Textbook of Medical Imaging. 7ª ed. Polônia: Elsevier, 2020; 2217p.

2. AIRAKSINEN O, et al. European guidelines for the management of chronic nonspecific low back pain. European Spine Journal, 2006; 15(Suppl 2):192-300. 
3. ALMEIDA DC, KRAYCHETE DC. Dor lombar - uma abordagem diagnóstica. Revista Dor, 2017 abr-jun; 18(2):173-7.

4. ALVES TCA, et al. Bloqueio peridural com corticoide em hérnia discal: revisão sistemática. Revista Neurociências, 2015; 23(4)575-80.

5. ANDERSON JAD. Problems of classification of low-back pain. Rheumatol Rehabilitation, 1977; 16(1):34-6.

6. BOONSTRA AM, et al. Reliability and validity of the visual analogue scale for disability in patients with chronic musculoskeletal pain. International Journal of Rehabilitation Research, 2008; $31(2): 165-9$.

7. CANNON DT, APRILL CN. Lumbosacral epidural steroid injections. Archive of Physical Medicine and Rehabilitation, 2000; 81(1):87-98.

8. COLLIGHAN N, GUPTA S. Epidural steroids. Continuing Education in Anaesthesia, Critical Care \& Pain, 2010; 10(1):1-5.

9. DENIS I, et al. Randomized Double-Blind Controlled Trial Comparing the Effectiveness of Lumbar Transforaminal Epidural Injections of Particulate and Nonparticulate Corticosteroids for Lumbosacral Radicular Pain. Pain Medicine, 2015; 16(1):1697-1708.

10. EL-YAHCHOUCHI C, et al. The Noninferiority of the Nonparticulate Steroid Dexamethasone vs the Particulate Steroids Betamethasone and Triamcinolone in Lumbar Transforaminal Epidural Steroid Injections. Pain Medicine, 2013; 14(11)1650-7.

11. GHARIBO C, et al. Epidural steroid injections: An update on mechanisms of injury and safety. Techniques In Regional Anesthesia \& Pain Management, 2009; 13(1):266-271.

12. HAWKER GA, et al. Measures of Adult Pain. Arthritis Care \& Research, 2011; 63(S11):240-52.

13. ITZ CJ, et al. Clinical course of non-specific low back pain: A systematic review of prospective cohort studies set in primary care. European Journal of Pain, 2013; 17(1):5-15.

14. JOHANSSON A, et al. Local corticosteroid application blocks transmission in normal nociceptor C-fibres. Acta Anaesthesiologica Scandinavica, 1990; 34(1):335-338.

15. KENNEDY DJ, et al. Comparative Effectiveness of Lumbar Transforaminal Epidural Steroid Injections with Particulate Versus Nonparticulate Corticosteroids for Lumbar Radicular Pain due to Intervertebral Disc Herniation: A Prospective, Randomized, Double-Blind Trial. Pain Medicine, 2014; 15(4):548-55.

16. LESLIE NG, et al. The efficacy of corticosteroids in periradicular infiltration for chronic radicular pain: a randomized, double-blind, controlled trial. Spine, 2005; 30(8):857-62.

17. MANSON NA, et al. Transforaminal epidural steroid injections prevent the need for surgery in patients with sciatica secondary to lumbar disc herniation: a retrospective case series. Canadian Journal of Surgery, 2013; 56(2):89-96.

18. MULLEMAN D, et al. Pathophysiology of disk-related sciatica. I._Evidence supporting a chemical component. Joint Bone Spine, 2006; 73(2):151-8.

19. O'DONNELL C, et al. Comparison of Triamcinolone to Dexamethasone in the Treatment of Low Back and Leg Pain via Lumbar Transforaminal Epidural Steroid Injection. The Spine Journal, 2008; 8(5):65-70.

20. PARK $\mathrm{CH}$, et al. Comparison of the Effectiveness of Lumbar Transforaminal Epidural Injection with Particulate and Nonparticulate Corticosteroids in Lumbar Radiating Pain. Pain Medicine, 2010; 11(11):1654-8.

21. PINHEIRO J, et al. Dor lombar crônica inespecífica e função: Estudo Clínico no Âmbito de uma Consulta de Medicina Física e de Reabilitação. Acta Médica Portuguesa, 2011; 24(S2):287-292.

22. POSTACCHINI F. Management of herniation of the lumbar disc. The Journal of Bone \& Joint Surgery (Br), 1999; 81(4):567-76.

23. RYDEVIK B, et al. Pathoanatomy and pathophysiology of nerve root compression. Spine, 1984; 9(1):7-15.

24. SAAL JA, et al. The natural history of lumbar intervertebral disc extrusions treated nonoperatively. Spine, 1990; 15(7):683-6.

25. SAAL JA, SAAL JS. Nonoperative treatment of herniated lumbar intervertebral disc with radiculopathy: an outcome study. Spine, $1989 ; 14(4): 431-7$.

26. SAYEGH FE, et al. Efficacy of steroid and nonsteroid caudal epidural injections for low back pain and sciatica: a prospective, randomized, double-blind clinical trial. Spine, 2009; 34(14):1441-7.

27. SCOTT J, HUSKISSON EC. Graphic representation of pain. Pain, 1976; 2(1):175-84.

28. SPACCARELLI KC. Lumbar and caudal epidural corticosteroid injections. Mayo Clinic Proceedings, 1996; 71(1):169178.

29. STUMP PRNAG, et al. Lombociatalgia. Revista Dor, 2016; 17(Suppl 1):63-6.

30. TONKOVICH-QUARANTA LA, WINKLER SR. Use of epidural corticosteroids in low back pain. The Annals of Pharmacotherapy, 2000; 34(1):1165-1172.

31. VIALLE LR, et al. Hérnia discal lombar. Revista Brasileira de Ortopedia, 2010; 45(1):17-22.

32. WHITE AA, PANJABI MM. Clinical biomechanics of the spine. 2nd ed. Philadelphia: JB Lippincott, 1990, 18p.

33. ZANON IB, et al. Comparison of the effectiveness of radicular blocking techniques in the treatment of lumbar disk hernia. Coluna/columna, 2015; 14(4):295-8. 